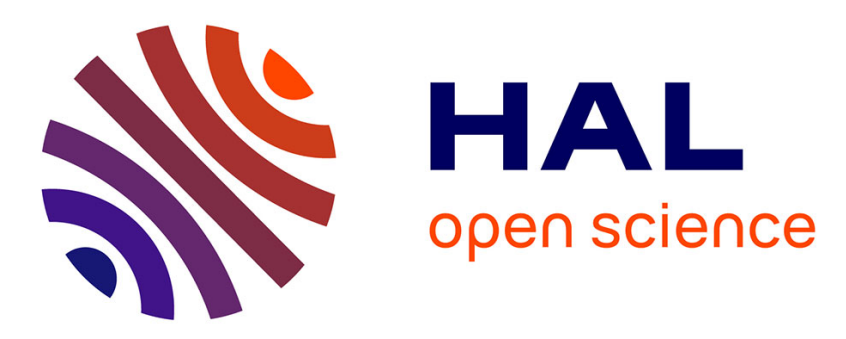

\title{
Mise en cohérence de données laser mobile sur un modèle cartographique par recalage non-rigide
}

Fabrice Monnier, Bruno Vallet, Nicolas Paparoditis, Jean-Pierre Papelard, Nicolas David

\section{- To cite this version:}

Fabrice Monnier, Bruno Vallet, Nicolas Paparoditis, Jean-Pierre Papelard, Nicolas David. Mise en cohérence de données laser mobile sur un modèle cartographique par recalage non-rigide. Revue Française de Photogrammétrie et de Télédétection, 2013, 202, pp.27 - 41. 10.52638/rfpt.2013.49 . hal-02552608

\section{HAL Id: hal-02552608 \\ https://hal.science/hal-02552608}

Submitted on 23 Apr 2020

HAL is a multi-disciplinary open access archive for the deposit and dissemination of scientific research documents, whether they are published or not. The documents may come from teaching and research institutions in France or abroad, or from public or private research centers.
L'archive ouverte pluridisciplinaire HAL, est destinée au dépôt et à la diffusion de documents scientifiques de niveau recherche, publiés ou non, émanant des établissements d'enseignement et de recherche français ou étrangers, des laboratoires publics ou privés. 


\title{
MISE EN COHÉRENCE DE DONNÉES LASER MOBILE SUR UN MODÈLE CARTOGRAPHIQUE PAR RECALAGE NON-RIGIDE.
}

\author{
Fabrice Monnier ${ }^{1}$, Bruno Vallet $^{1}$, Nicolas Paparoditis $^{1}$, Jean-Pierre Papelard $^{1}$, Nicolas David $^{1}$ \\ 1: IGN/SR, MATIS, Université Paris-Est, 73 avenue de Paris, 94160 Saint Mandé, France
}

\begin{abstract}
Résumé
Depuis quelques années, des véhicules de cartographie mobile ont été développés pour acquérir des données géoréférencées très précises et en grande quantité au niveau du canyon urbain. L'application majeure des données collectées par ces véhicules est d'améliorer les bases de données géographiques existantes, en particulier leur précision, leur niveau de détail et la diversité des objets représentés. On peut citer entre autres applications la modélisation géométrique fine et la texturation des façades, l'extraction de "petits" objets comme les troncs d'arbres, poteaux, panneaux, mobiliers urbain, véhicules,... Cependant, les systèmes de géopositionnement de ces véhicules ne parviennent pas à fournir une localisation d'une précision suffisante pour cette tâche. En particulier, les masques GPS fréquents en milieu urbain sont paliés par les mesures de la centrale inertielle grâce à un algorithme de fusion de données pouvant entraîner une dérive. C'est pourquoi, un recalage est indispensable pour mettre en correspondance ces données mobiles très détaillées avec les bases de données géographiques moins détaillées mais mieux géopositionnées, qu'elles soient 2D ou 3D. Cet article présente une méthode générique et efficace permettant un tel recalage. Le processus est basé sur une méthode de type ICP ("Iterative Closest Point") point à plan. On suppose que l'erreur de géopositionnement, ou dérive varie de façon non linéaire, mais lentement en fonction du temps. On modélise donc la trajectoire par une "chaîne" ayant une certaine rigidité. A chaque itération, la trajectoire est déformée afin de minimiser la distance des points laser aux primitives planes du modèle. Cette méthode permet d'approximer la dérive par une fonction linéaire par intervalle de temps. La méthode est testée sur des données réelles ( 3,6 millions de points laser acquis sur un quartier de la ville de Paris sont recalés sur un modèle 3D d'environ 71.400 triangles). Enfin, la robustesse et la précision de cet algorithme sont évaluées et discutées.
\end{abstract}

Mots clés : Recalage non-rigide, ICP, Modèle géométrique 3D, Données laser mobiles.

\begin{abstract}
Over the last years, a growing number of mobile mapping systems have been developped in order to obtain large amounts of accurate georeferenced data on urban canyons. The main application of such data is to improve the existing geographic database, particularly in terms of its accuracy, the level of detail and the diversity of represented objects. Indeed, we should mention fine geometric modelling and fine façade texturation, object extraction such as trees, poles, road signs marks, furniture, vehicles, etc. However, the geopositionning system fails to give sufficient positionning accuracy for that purpose. In particular, GPS masks are corrected by a Inertial Navigation System (INS) which can induce drifts. That is why it is absolutly necessary to register the mobile data with a high level of detail but with poor positionning and a low level of detail of the geometric database but which is accurately localised, whether in $2 D$ or $3 D$. This article presents a generic and efficient method of such registration. The process is based on a method like a point to plane ICP ("Iterative Closest Point"). According to the georeferencing error, the drift vary non-linearly, but slowly in time, the trajectory will be model as a chain with a certain rigidity. For each iteration, the trajectory is deformed in order to minimise the distance between laser points and planar model primitives. Thus, the drift is approximated by a piecewise linear function of time. The method has been tested on real data (a scan of the city of Paris at 3.6 million laser points on a 3D model of approximately 71,400 triangles). Finally, the robustness and the accuracy of this algorithm are evaluated and discussed.
\end{abstract}

Keywords : Non-rigid registration, ICP, 3D Geometric model, Mobile laser scans.

\section{Introduction}

\subsection{Contexte général}

L'institut national de l'information géographique et forestière (IGN) a pour vocation de décrire précisément la surface du territoire national français. Pour ce faire, l'IGN a acquis des compétences dans le domaine de la cartographie, de l'imagerie (aérienne ou terrestre) et de la photogrammétrie. Grâce à cela, il dispose aujourd'hui d'une quantité importante de données images (terrestres, aériennes et satellitaires), d'acquisitions lidar (terrestres ou aériennes) et de bases de données géographiques $2 \mathrm{D}$ ou $3 \mathrm{D}$.
Dans ce contexte, il apparaît indispensable de recaler ces données entre elles, en particulier pour les applications suivantes:

- détecter des changements pour la mise à jour de bases de données géographiques;

- enrichir les bases de données géographiques existantes en augmentant significativement leur niveau de détail (géométrie et texturation fines);

- recaler des données brutes entre elles pour que les informations qui en sont extraites soient directement exploitables dans un référentiel commun. 
Le travail présenté ici s'intéresse plus particulièrement au problème du recalage de données de cartographie mobiles sur des bases de données géographiques 2D ou 3D.

\subsection{Les modèles}

Dans cet article, nous appelons modèle toute base de données géographiques, qu'elles soient 2D ou 3D.

Un modèle est composé de primitives géométriques qui peuvent être :

- ponctuelles (0D): repères de nivellement, sommets d'objets polyédriques, position de troncs d'arbres ou de poteaux en $2 \mathrm{D}, \ldots$

- linéaires (1D): arêtes d'objets polyédriques, linéaires 2D (cadastre, base de données topographiques ou parcellaires en représentation 2D, limites de trottoirs,...).

- surfaciques (2D): faces d'objets polyédriques.

Ces modèles généralisent l'environnement, dans le sens où ils représentent grossièrement la réalité. Ils ont donc un niveau de détails sommaire (de l'ordre du mètre). Par contre, leur géopositionnement est très précis car ils sont construits sur des points mesurés par des opérateurs.

\subsection{Les données mobiles}

Dans cet article, nous appelons données mobiles toute donnée (image ou laser) acquise par un système de cartographie mobile. Un système de cartographie mobile est un véhicule embarquant:

- des capteurs image et/ou laser percevant l'environnement;

- un système de positionnement permettant de géolocaliser les données acquises par ces capteurs.

Les systèmes de ce type permettent de rapprocher le capteur de la donnée à observer. Le niveau de détail de ces acquisitions est donc accru par rapport à l'imagerie aérienne. Cet avantage a conduit à un essor rapide de ce type de systèmes au cours de ces dernières années.

Le système de géopositionnement d'un véhicule de cartographie mobile se compose généralement:

- d'un système GPS permettant de capter la position avec une précision variable en fonction des conditions d'acquisition et du système allant de quelques mètres à moins de $10 \mathrm{~cm}$,

- d'un odomètre (parfois) mesurant la distance parcourue par le véhicule,

- d'une centrale inertielle composée d'accéléromètres mesurant l'accélération du véhicule, de gyromètres mesurant l'accélération angulaire et de magnétomètres pour obtenir à tout instant la position du nord géographique dont la précision dépendant du système utilisé.

Les données issues de ces capteurs sont intégrées afin de déduire la position précise du véhicule à chaque instant. Cependant, ce géoréférencement peut-être perturbé par:
- les trajets multiples: le capteur GPS reçoit plusieurs fois le même signal, soit directement, soit indirectement (reflété par des façades par exemple) ce qui le perturbe,

- les masques GPS: la portion de ciel visible est trop faible et le capteur ne voit plus assez de satellites pour en déduire sa position.

Ces perturbations entraînent une erreur absolue sur l'estimation de la trajectoire du véhicule qui n'est que partiellement compensée par les informations de la centrale inertielle. Cette compensation partielle induit en général un écart qui peut atteindre plusieurs mètres dans le cas d'un masque GPS de plusieurs minutes. Dans cet article, nous appelons dérive cet écart entre la trajectoire fournie par le système de géopositionnement et la trajectoire réelle (idéale). Le recalage effectué va chercher à estimer cette dérive. La dérive dépend non seulement de la qualité des données de positionnement mais aussi de l'algorithme d'intégration des données des capteurs de positionnement, et doit donc être considérée comme non linéaire en fonction du temps (il peut y avoir dérive même quand le véhicule est à l'arrêt). L'utilisation d'une centrale inertielle de bonne qualité garantit cependant une variation très lente de la dérive ainsi qu'une très bonne orientation du véhicule. Les dérives sont donc principalement composées de dérives lentes en translation. C'est sur ces deux hypothèses que se base notre recalage et en particulier notre modèle de dérive.

\subsection{Problématique}

Dans cet article, nous nous intéressons à l'utilisation d'un modèle (au sens de la partie 1.2) pour recaler des données laser mobile (au sens de la partie 1.3). La motivation sous-jacente est double:

- combiner la précision du modèle (peu détaillé) avec le niveau de détail des données mobiles (moins précises),

- mettre le modèle et les données mobiles en cohérence afin de pouvoir exploiter ces données mobiles pour améliorer la géométrie du modèle, mais aussi sa texture puisque si le véhicule est équipé de caméras, elles bénéficieront aussi du recalage des données laser sur le modèle.

La méthode décrite dans cet article peut s'appliquer à tout système de géopositionnement pour lequel les variations de la dérive sont suffisamment lentes (ce qui est au moins le cas de tous les systèmes intégrant une centrale inertielle ou des accéléromètres suffisamment précis).

Les verrous majeurs à ce recalage sont:

- le niveau de détail incompatible entre la base de données (de l'ordre du mètre au mieux) et le nuage laser (centimétrique),

- la nature fortement non linéaire en fonction du temps de l'erreur de positionnement du véhicule d'acquisition.

L'objectif principal n'est pas d'obtenir un géoréférencement absolu centimétrique des données acquises, mais d'assurer leur mise en cohérence relative pour les rendre exploitables. 


\section{Etat de l'art}

Le recalage de données est une thématique abordée depuis des années par différentes communautés scientifiques (vision par ordinateur, photogrammétrie, imagerie médicale, intelligence artificielle,...). L'objectif principal consiste à mettre en cohérence au minimum deux jeux de données entre eux. Généralement, l'un des jeux de données sert de référence sur lequel le second est recalé. Le but est donc de déterminer la transformation (rotation, translation) nécessaire pour rapprocher au mieux les données entre elles. Pour cela, on peut schématiser grossièrement une technique de recalage comme étant composée de deux étapes importantes. La première consiste à effectuer une comparaison entre les jeux de données afin d'extraire des caractéristiques communes dans chacun d'eux. La seconde, utilise ces caractéristiques afin de déterminer la transformation optimale à appliquer. Ces méthodes, résumées dans (Gressin et al., 2013) fonctionnent tant de manière rigide que non rigide sur des problématiques $2 \mathrm{D}$ comme $3 \mathrm{D}$ avec des méthodes point à point ou encore point à surface. La technique utilisée est complètement dépendante des données que l'on possède au départ et des informations qu'elles sont capables de fournir. La technique de référence à l'heure actuelle et qui reste la plus utilisée (Salvi et al., 2007) pour le recalage de données est l'ICP ou "Iterative Closest Point". Cette méthode de recalage a l'avantage d'être simple à appréhender tout en donnant de très bons résultats. Son inconvénient est de devoir disposer d'une bonne estimation de la dérive (transformation) de départ ou autrement dit de disposer d'une bonne initialisation des données (Chen et Medioni, 1992). Elle fonctionne de manière itérative et consiste à minimiser une énergie jusqu'à convergence afin de déterminer la transformation optimale qui met en correspondance les ensembles à recaler. L'ICP a été introduite pour la première fois par (Besl et McKay, 1992) et procédait par la mise en correspondance d'entités entre deux ensembles initiaux de nature similaire ou différente. Cette mise en correspondance était faite de manière simple par appariement des points les plus proches entre eux dans les deux jeux de données. Depuis, de nombreuses recherches ont été effectuées pour améliorer cette méthode. Certains auteurs comme (Rusinkiewicz et Levoy, 2001) distinguent six étapes de l'ICP décrites ci-dessous.

\section{Sélection des points :}

Lorsque l'on souhaite utiliser des techniques de mise en correspondance de ce type, il est nécessaire d'extraire des caractéristiques communes dans les jeux de données pour pouvoir ensuite les apparier. Cette étape est de loin la plus importante car elle détermine la qualité du futur recalage. Certaines caractéristiques permettent de se rendre invariant en rotation, translation et changement d'échelle (Sharp et al., 2002). Cela permet une grande robustesse au bruit ("outliers") et de se rendre, suivant la méthode utilisée, indépendant de l'initialisation de la donnée. Ces caractéristiques communes sont souvent des primitives. Elles peuvent être ponctuelles, linéaires, surfaciques ou d'ordre supérieur et de natures variées. Les primitives ponctuelles ont été beaucoup utilisées comme les points "SIFT" (Huber et Hebert, 2003) et (Barnea et Filin, 2008), les images de spin (Johnson et Hebert, 1999) et les coins (Thirion,
1996). Les primitives linéaires ont été utilisées comme les segments ou les courbes dans (Stein et Medioni, 1992) ou encore la transformée de Hough (Hecker et Bolle, 1994) et surfaciques comme les plans (Dold et Brenner, 2006). Certains auteurs utilisent des descripteurs de forme comme (Hahnel et al., 2003) et (Zheng et al., 2010) qui utilisent des méthodes de morphologie mathématique pour définir un squelette des objets. D'autres comme (Gelfand, 2005) ou (Demantké et al., 2012) ont mis en place des descripteurs locaux dit géométriques. Les auteurs de (Al-Durgham et al., 2010) utilisent l'un des nuages de points pour effectuer un maillage (triangulation de Delaunay). Cela permet de modifier l'appariement des points et de minimiser un volume au lieu d'une distance. Peu importe la méthode utilisée, elles ont toutes l'avantage de réduire de façon significative le nombre de points sur lesquels déterminer la transformation. Un autre avantage de ces techniques est qu'elles peuvent être ensuite associées à des méthodes de recalage tant simultanées (directe) que séquentielles (itératives).

\section{Appariement des points sélectionnés :}

Lorsque les caractéristiques ont été déduites dans chacun des jeux de données, des paires homologues peuvent être créées. Une paire définit un appariement et certains auteurs comme (Gelfand et al., 2003) utilisent une sélection précise et stratégique des appariements. Les auteurs de (Rusinkiewicz et Levoy, 2001) et (Zhang, 1994) font une étude sur les différents types d'échantillonnage de départ et montrent qu'un échantillonnage par les normales est plus robuste qu'un échantillonnage régulier ou aléatoire. De plus, cette étape étant en générale coûteuse en temps de calcul, elle peut être améliorée en utilisant des techniques d'échantillonnage spatial de type kd-tree (Zhang, 1994) et (Eggert et Dalyot, 2012) qui permettent éventuellement le passage à l'échelle lors de l'utilisation de grande quantité de données.

\section{Acceptation ou rejet des appariements :}

Cette étape permet de rejeter de mauvais appariements. Elle est en générale basée sur un seuil de distance séparant les points constitutifs des paires homologues.

\section{Pondération des appariements :}

Dans certains cas, tout les appariements ont le même poids (Besl et McKay, 1992) mais il peut s'avérer utile de pondérer les appariements. En général, ce poids peut être déduit par une gaussienne en fonction de la distance séparant les appariements ou encore par un critère de compatibilité des normales (Zhang, 1994).

\section{Définition et Minimisation d'une erreur métrique :}

Cette étape permet de déterminer la transformation (Rotation,Translation) optimale pour recaler les jeux de données entre eux. Elle peut être de plusieurs natures comme des processus linéaires basés sur l'utilisation des moindres carrés ou plus complexes : les auteurs de (Fitzgibbon, 2003), par exemple, utilisent une méthode non linéaire de minimisation (Levenberg-Marquardt) pour affiner les valeurs de la transformation ainsi déduite. 
Toutefois, d'autres méthodes que l'ICP existent pour recaler des données entre elles. On peut citer (Pottmann et al., 2004) qui utilisent des approximations quadratiques locales, (Ripperda et Brenner, 2005) qui utilise des techniques basées sur l'étude des distributions des normales nommées NDT pour "Normal Distribution Transform", (Tsin et Kanade, 2004) qui effectuent leur recalage en utilisant des noyaux de corrélation, (Chen et al., 1999) qui utilisent "RANSAC" (Random Sample Concensus), (Jian et Vemuri, 2005) qui intègrent des mélanges de gaussiennes, (Wolfson et Rigoutsos, 1997) qui utilisent une méthode de recalage basée sur du hachage géométrique ou encore (Tarel et Boujemaa, 1995) qui utilise une méthode floue de recalage.

Positionnement Les a priori sur la dérive évoqués dans la partie 1.3 nous permettent de nous orienter vers une méthode de recalage de type ICP. Cependant, nous avons aussi vu que la dérive est non-linéaire en fonction du temps. La stratégie de départ est donc d'utiliser une méthode simple et robuste de recalage se basant sur l'ICP entre les primitives géométriques du modèle et les points des données laser mobiles. Cette technique de mise en correspondance sera caractérisée par un modèle de dérive non rigide défini linéairement par intervalles de temps.

La contribution principale de l'article par rapport à l'état de l'art est la définition de ce modèle de dérive particulièrement bien adapté aux systèmes d'acquisition de cartographie mobile et donc à la dérive que l'on cherche à estimer. Les autres particularités de notre méthode sont:

- une sélection des points à l'aide d'un descripteur géométrique local s'inspirant de (Demantké et al., 2012).

- l'utilisation de la géométrie d'acquisition pour définir une compatibilité entre les normales du modèle et du nuage. Cette compatibilité permet une plus grande robustesse dans l'appariement comme démontré par (Rusinkiewicz et Levoy, 2001).

- une étape d'appariement accélérée (points laser / primitives géométriques du modèle) à l'aide d'un KdTree (Rusinkiewicz et Levoy, 2001) et (Zhang, 1994).

\section{Modèle de dérive}

Nous allons maintenant présenter les choix faits pour modèliser la dérive $D$.

\subsection{Choix du modèle}

Comme expliqué dans la partie 1.3, notre choix de modèle est guidé par trois caractéristiques des dérives des systèmes de positionnement:

- la lenteur de la variation de la dérive,

- la qualité de l'orientation (dérive en rotation négligeable par rapport à la dérive en translation),
- la dérive est indépendante de la vitesse du véhicule (il y a dérive même lorsque le véhicule est à l'arrêt).

Ainsi, nous avons choisi un modèle de dérive défini par une translation linéaire par morceaux en fonction du temps $t$. Le choix de la dépendance temporelle est naturel au vu de la troisième caractéristique, et permet en plus de gérer la différence de dérive du véhicule aux recoupements (passage au même endroit à des instants différents). Enfin, le choix d'une interpolation linéaire plutôt que d'un découpage en blocs rigides (Gressin et al., 2013) permet d'estimer la dérive de façon beaucoup plus fine.

\subsection{Formalisme}

La dérive de la trajectoire de départ s'écrit donc comme une translation $D: t \longrightarrow \mathbb{R}^{3}$ qui permet de définir la trajectoire recalée du véhicule:

$$
P_{\text {rec }}=P_{\text {init }}+D(t)
$$

où $P_{\text {init }}$ est la position initiale d'un point du nuage (calculée par le système de positionnement), $P_{r e c}$ est sa position après recalage et $t$ son instant acquisition. Cette information est donnée par le GPS avec une précision de l'ordre du centième de seconde.

Cette dérive $D$ est discrétisée par une fonction linéaire par morceaux. Pour un point $P_{\text {init }}$ acquis au temps $t$, le point $P_{\text {rec }}$ correspondant sera déterminé en interpolant $P_{\text {init }}$ (cf.équation 2) entre des valeurs de dérive $\delta_{c}$ données à des temps de contrôle $T_{c}$ espacés régulièrement d'un intervalle de temps $\Delta_{t}$ (cf.Figure 1):

$$
\begin{gathered}
D_{\delta}(t)=\frac{\left(T_{c^{+}(t)}-t\right) \delta_{c^{-}(t)}+\left(t-T_{c^{-}(t)}\right) \delta_{c^{+}(t)}}{\Delta_{t}}= \\
(1-\alpha(t)) \delta_{c^{-}(t)}+\alpha(t) \delta_{c^{+}(t)}
\end{gathered}
$$

Où les variables connues au temps $t$ sont :

$c^{+}(t)$ : Indice du premier temps de contrôle supérieur à $t$.

$c^{-}(t)$ : Indice du premier temps de contrôle inférieur à $t$.

$\alpha(t)=\left(t-T_{c^{-}(t)}\right) / \Delta_{t}$ : Coefficient d'interpolation.

Les inconnues définissant la dérive sont :

$\delta_{c^{+}(t)}$ : Dérive au temps de contrôle $T_{c^{+}(t)}$.

$\delta_{c^{-}(t)}$ : Dérive au temps de contrôle $T_{c^{-}(t)}$.

Le pas de discrétisation temporelle $\Delta_{t}$ doit être choisi assez petit pour pouvoir représenter la non linéarité de la dérive avec une précision suffisante, ce qui sera discuté dans la partie 5.2.1.

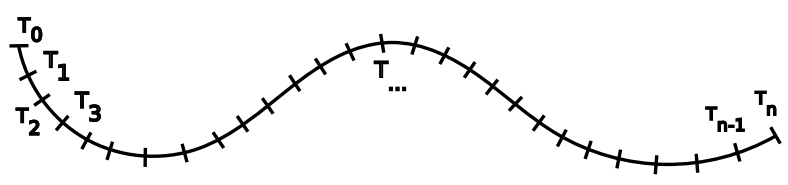

Figure 1: Découpage de la trajectoire en temps de contrôle.

Les inconnues déterminées par ICP sont donc les trois composantes des vecteurs $\delta_{c}$ qui définissent la dérive par l'équation (2). Cela permet d'obtenir la nouvelle la trajectoire 
du véhicule par l'équation (1) et donc le nuage recalé complet par application de la dérive à chaque point.

De manière générale, dans cet article, une trajectoire recalée $T r a j_{r e c}$ sera donc toujours définie par $T r a j_{r e c}=$ $\operatorname{Traj}_{\text {init }}+D_{\delta}(t)$ où $T r a j_{i n i t}$ est la trajectoire de départ et $\delta$ le vecteur concaténant les dérives $\delta_{c}$ aux temps de contrôle $T_{c}$ et qui le définissent donc de façon différentielle par rapport aux données mobiles initiales (cf Figure 2). Cela nous permet notamment de définir une distance simple entre deux trajectoires définies par $\delta^{i}$ et $\delta^{j}$ que nous appellerons dérive moyenne:

$$
D M\left(\delta^{i}, \delta^{j}\right)=\frac{1}{N_{T c}} \sum_{c=1}^{N_{T c}}\left\|\delta_{c}^{i}-\delta_{c}^{j}\right\|
$$

où $N_{T c}$ représente le nombre de $T c$ le long de la trajectoire. Cela permet de prendre en compte non seulement la géométrie des nuages mais aussi la dimension temporelle puisque les distances sont mesurées entre points acquis au même instant (aux temps de contrôle $T_{c}$ ) et non entre les points les plus proches des deux nuages. Notons aussi que cette distance peut être vue à la fois comme une distance entre les nuages et entre les trajectoires correspondantes puisque la dérive $D_{\delta}(t)$ s'applique aux deux.

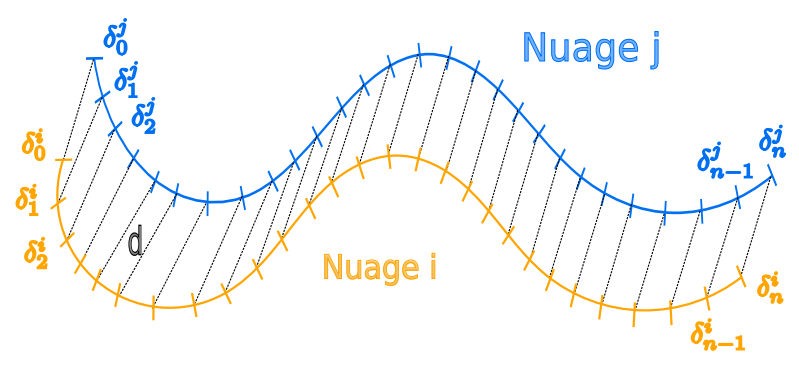

Figure 2: Illustration de la distance $d$ séparant les dérives $\delta_{c}^{n}$ aux différents temps de contrôle $T_{c}$ entre deux données mobiles pour le calcul de la dérive moyenne $D M$.

\subsection{Rigidité}

Comme ce modèle est déformable, il faut paramétrer sa rigidité. Nous faisons cela en définissant une énergie de déformation correspondant, par analogie avec une déformation mécanique, à la quantité d'énergie à apporter au système pour lui appliquer cette déformation (en particulier elle doit être nulle pour une transformation rigide). Nous allons donc définir une énergie de déformation:

$$
E_{\text {def }}(\delta)=\sum_{c=1}^{N b_{T c}-1}\left\|\delta_{c+1}-\delta_{c}\right\|^{2}
$$

Cette énergie traduit le fait que la dérive au temps $T_{c}$ doit être proche de celle au temps $T_{c+1}$, ce qui permet d'introduire l' $a$ priori mentionné en partie 1.3: la variation de la dérive est lente. Elle aidera aussi la convergence en propageant les contraintes le long de la trajectoire.

\section{ICP (Iterative Closest Point)}

\subsection{Principe Général}

L'ICP est une méthode de recalage fonctionnant par mise en correspondance des jeux de données de manière séquen- tielle (itérative). Chaque itération doit rapprocher les jeux de données entre eux par minimisation d'une énergie $E_{\text {reg }}$. Le processus itératif est stoppé lorsqu'un critère d'arrêt est atteint. Généralement, l'un des jeux de données sert de référence sur lequel le second vient s'appuyer. Le point fort de cette méthode est qu'elle peut s'adapter à différents types de support comme:

- recalage point / point,

- recalage point / primitive,

- recalage primitive / primitive.

L'inconvénient majeur est le besoin de disposer au départ de valeurs approchées des paramètres de la dérive (rotation et translation) mais notre système d'acquisition assure une initialisation correcte des données laser.

\subsection{Les différentes étapes}

Nous reprenons le découpage classique de l'ICP en six étapes (cf.Partie 2).

\subsubsection{Sélection}

La sélection des points à utiliser pour le recalage est une étape essentielle de la méthode. Dans notre étude, les modèles utilisés décrivent la géométrie des bâtiments en contexte urbain par le biais de primitives géométriques 3D (triangles). Pour effectuer un recalage, il est donc nécessaire de déterminer quels points dans un nuage laser correspondent à des faces de bâtiment afin de n'apparier qu'eux aux faces du modèle. Dans nos expérimentations, le sol n'est pas scanné, et les toits ne sont en général pas visibles depuis le sol, donc nous nous sommes limités à la sélection de points de façades. Il serait possible de travailler en 2D, mais nous avons choisi de rester en 3D. Cela permet, d'une part, de pouvoir étendre la méthode simplement aux scans observant aussi le sol (correction altimétrique) et, d'autre part, de traiter certains cas particuliers comme un petit bâtiment devant un plus grand ou des décrochages verticaux de façades.

La sélection des points de façade se fait par seuillage d'un descripteur géométrique local calculé en chaque point laser 3D. Ce descripteur, introduit par (Demantké et al., 2012), décrit la probabilité d'un point laser $P$ d'appartenir à une façade (cf.Figure 3).

La première étape de cet algorithme est d'analyser la géométrie locale au voisinage d'un point laser en effectuant une analyse en composante principale $(A C P)$. Pour chaque point laser $P$ le tenseur de structure 3D (ellipsoïde) est calculé autour d'un groupe de voisins $V_{P}$ dont la taille est déterminée par un critère entropique. Cet ellipsoïde est défini par trois vecteur orthogonaux classés par ordre décroissant. Notés $\overrightarrow{e_{1}}, \overrightarrow{e_{2}}$ et $\overrightarrow{e_{3}}$ et de normes respectives $\sigma_{1}, \sigma_{2}, \sigma_{3}$, ils représentent la répartition spatiale du groupe $V_{P}$. La normale au point $P$ est donné par $\overrightarrow{e_{3}}$ et permet de définir un score de verticalité $S_{\text {vertical }}$ :

$$
S_{\text {vertical }}=1-\left|z_{\overrightarrow{3}}\right| \in[0,1] .
$$

Une seconde caractéristique, la "planarité" $S_{\text {plan }}$, peut être déduite de ce tenseur. Il mesure l'applatissement du tenseur dans le plan $\left(\overrightarrow{e_{1}}, \overrightarrow{e_{2}}\right)$ perpendiculaire à $\overrightarrow{e_{3}}$ et est défini comme :

$$
S_{\text {plan }}=\frac{\sigma_{2}-\sigma_{3}}{\sigma_{1}} \in[0,1] .
$$


En combinant ces deux caractéristiques, on peut définir un descripteur géométrique local traduisant la probabilité d'un point $P$ d'appartenir à une façade (plan vertical):

$$
S_{\text {facade }}=S_{\text {plan }} \times S_{\text {vertical }} \in[0,1] .
$$

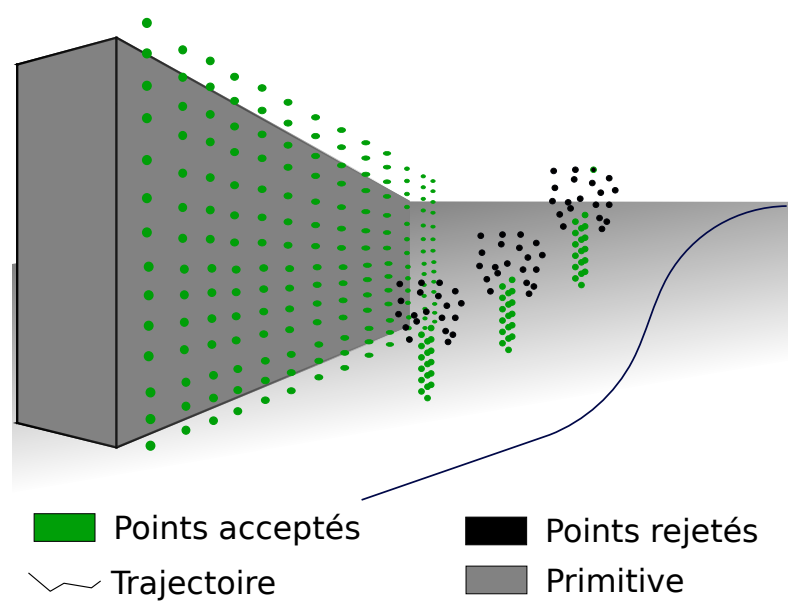

Figure 3: Sélection des points de façade par un descripteur géométrique local adapté.

\subsubsection{Appariement et acceptation.}

Les points de "façade" étant sélectionnés, il est dorénavant possible de les apparier aux primitives de notre modèle. On dira qu'un appariement est bon si le point des données provient d'un écho laser sur un objet réel que la primitive appariée représente. Dans tous les autres cas, on dira que l'appariement est mauvais. Les mauvais appariements ont principalement deux sources:

- une dérive suffisante pour qu'un point soit attribué à la mauvaise primitive,

- l'existence d'objets non modélisés dans la scène, et donc de points ne correspondants pas à une primitive du modèle. Dans notre cas, l'étape de sélection réduit le nombre de points de ce type mais ne peut pas les éliminer complètement.

Les mauvais appariements peuvent induire de larges erreurs sur l'estimation de la dérive s'ils deviennent majoritaires sur les bons. Ainsi, l'objectif est de générer le plus possible de bons appariements et le moins possible de mauvais. Pour cela, les points sont généralement appariés aux primitives les plus proches à condition que la distance du point à la primitive soit inférieure à un seuil fixé $d_{\max }$.

Dans notre cas, le système d'acquisition mobile utilisé enregistre la position spatiale du centre du laser, noté $C$, pour chaque point $P$, ce qui permet notamment:

- de définir le rayon laser $R$ entre le centre au laser $C$ et le point $P$ correspondant.

- de définir l'orientation de la normale du nuage au point $P$. Dans notre étude, la direction de la normale $\vec{n}_{P}$ est calculée en utilisant les travaux de (Demantké et al., 2012), et est orientée en direction du centre du laser.
Chaque primitive Prim du modèle géométrique utilisé ayant sa normale $\vec{n}_{\text {Prim }}$ orientée de l'intérieur vers l'extérieur, nous pouvons affiner l'acceptation des appariements en rajoutant un critère de compatibilité des normales. Enfin, nous utilisons le rayon $R$ pour apparier un point non plus à la primitive la plus proche mais à la première primitive intersectée par ce rayon. Plus précisément, un point $P$ est apparié à une primitive Prim seulement si: Prim est intersectée par le rayon $R$ (cf.Figure 4) et qu'elle vérifie trois conditions:

1. compatibilité des normales: $\vec{n}_{\text {Prim }} \cdot \vec{n}_{P}>0$

2. distance d'acceptation: $\operatorname{dist}(P, \operatorname{Prim})<d_{\max }$ (cf.Figure 5).

3. si plusieurs primitives sont intersectées dans la zone d'acceptation, la plus proche est sélectionnée.

Ainsi, ces informations supplémentaires permettent:

- d'améliorer la robustesse des appariements grâce à l'apport de la compatibilité des normales, et donc la robustesse de l'ICP.

- d'utiliser un lancé de rayon pour sélectionner les primitives candidates à l'appariement. En effet, l'appariement est de loin l'étape la plus coûteuse en temps d'exécution en particulier lorsque le nombre de primitives est important. Le lancé de rayon a l'avantage d'être une technique classique donc très optimisée pour laquelle des librairies performantes peuvent facilement être utilisées pour accélérer l'appariement.

Notons toutefois que si l'information du centre au laser n'est pas disponible, la méthode présentée reste utilisable en revenant à un appariement plus classique, au seul coût de perdre ces deux avantages.

Dans le reste de cet article, nous indicerons les appariements par $i$. Ainsi pour un appariement $i$ correspond:

- le point laser apparié $P_{i}$, acquis au temps $t_{i}$

- la primitive $\operatorname{Prim}_{i}$ appariée à $P_{i}$.

- le centre au laser $C_{i}$ associé à $P_{i}$.

- le rayon laser $R_{i}$.

- la normale $\vec{n}_{P_{i}}$ associée à $P_{i}$.

- la normale $\vec{n}_{\text {Prim }_{i}}$ associée à $\operatorname{Prim}_{i}$.

\subsubsection{Pondération des appariements}

Pour la pondération des appariements, nous utilisons encore un critère de compatibilité des normales. Elle est définie par:

$$
w_{i}=\vec{n}_{P_{i}} \cdot \vec{n}_{\text {Prim }_{i}}
$$

qui est forcément positive grâce à la sélection qui rejette les appariements pour lesquels $w_{i} \leq 0$ et correspond au cosinus de l'angle entre les normales. 


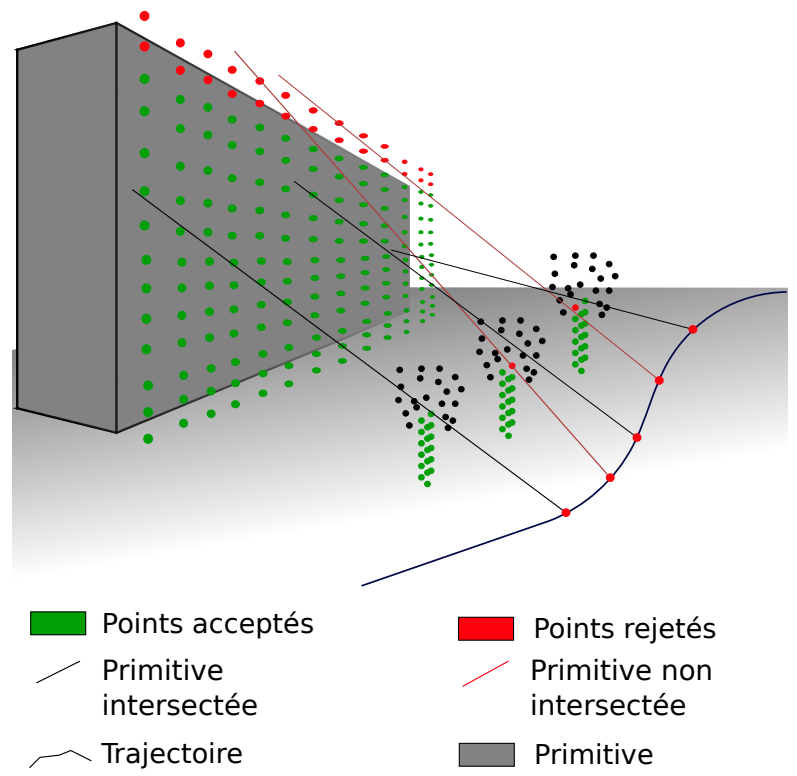

Figure 4: Illustration de l'étape d'appariement des points laser 3D aux primitives géométriques par lancé de rayon.

\subsubsection{Attache au modèle}

Chaque itération de l'ICP cherche à rapprocher les données du modèle. Cela se fait par minimisation d'un terme d'attache au modèle. Dans notre cas, nous pouvons simplement le définir comme:

$$
\begin{array}{r}
E_{\text {model }}\left(\delta_{c}\right)=\sum_{i=1}^{N_{a p p}} w_{i} \cdot \operatorname{dist}\left(P_{i}(\delta), \operatorname{Prim}_{i}\right)^{2}= \\
\sum_{i=1}^{N_{a p p}} w_{i} \cdot\left(\left(P_{i}+D_{\delta}\left(t_{i}\right)-Q_{i}\right) \cdot \vec{n}_{\text {Prim }_{i}}\right)^{2}
\end{array}
$$

Où $P_{i}(\delta)=P_{i}+D_{\delta}\left(t_{i}\right)$ est un point apparié auquel on a appliqué la dérive définie par $\delta$ (cf.équation 2), $N_{a p p}$ est le nombre total d'appariements et $Q_{i}$ un point appartenant à la primitive $\operatorname{Prim}_{i}$ obtenu par projection orthogonale.

\subsubsection{Minimisation}

Le problème de recalage (pour un appariement donné) consiste à trouver la dérive $D_{\delta}(t)$ définie par l'ensemble des $\delta_{c}$ qui minimise:

$$
E_{\text {reg }}(\delta)=E_{\text {model }}(\delta)+\lambda_{\text {rigid }} * E_{\text {def }}(\delta)
$$

avec $\lambda_{\text {rigid }}$ un paramètre de rigidité choisi par l'utilisateur (cf.partie 5.2.2).

Nous allons maintenant écrire (10) sous forme matricielle, en séparant les deux énergies. D'après l'équation (2) chaque terme de $E_{\text {model }}$ mis au carré dans (9) est :

$\operatorname{dist}\left(P_{i}\left(\delta_{c}\right), \operatorname{Prim}_{i}\right)=$

$$
\begin{aligned}
\left(1-\alpha\left(t_{i}\right)\right) \delta_{c^{-}\left(t_{i}\right)} \cdot \vec{n}_{\operatorname{Prim}_{i}}+\alpha\left(t_{i}\right) \delta_{c^{+}\left(t_{i}\right)} \cdot \vec{n}_{\text {Prim }_{i}} \\
-\left(Q_{i}-P_{i}\right) \cdot \vec{n}_{\text {Prim }_{i}}
\end{aligned}
$$

$E_{\text {model }}$ peut donc s'écrire sous forme matricielle (moindres carrés pondérés):

$E_{\text {model }}(\delta)=\left(A_{\text {model }} \delta-b_{\text {model }}\right)^{t} W_{\text {model }}\left(A_{\text {model }} \delta-b_{\text {model }}\right)$

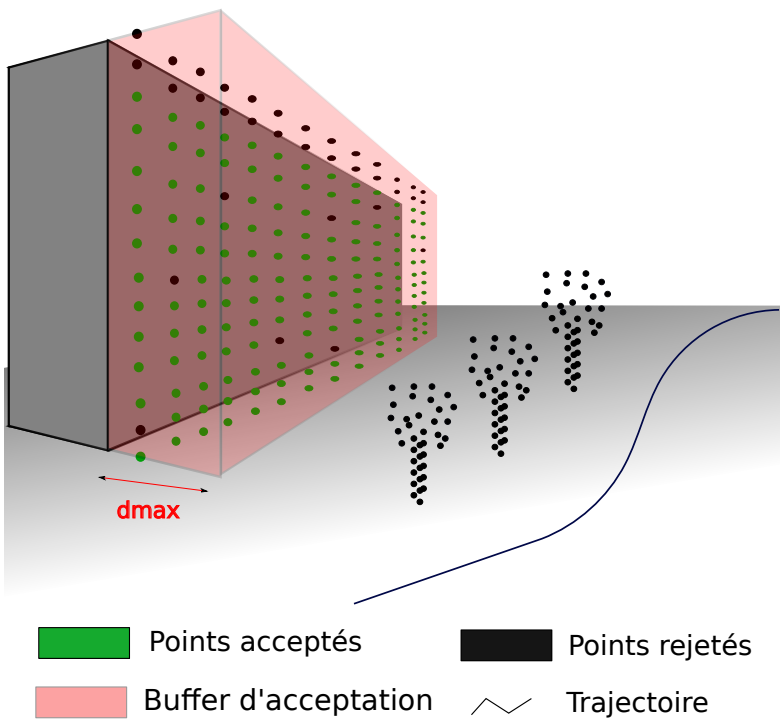

Figure 5: Illustration de l'étape de sélection des points appariés en fonction de leur distance aux primitives géométriques.

où:

- $\delta=\left(\delta_{1}^{x}, \delta_{1}^{y}, \delta_{1}^{z}, \delta_{2}^{x}, \ldots, \delta_{N_{T_{c}}}^{z}\right)^{t}$ est un vecteur de dimension $\left(3 N_{T_{c}}\right)$ sauvegardant la concaténation des vecteurs de dérive $\delta_{c}$ en chaque temps de contrôle $T_{c}$

- $b_{\text {model }}$ est un vecteur dimension $N_{a p p}$ dont le $i^{\text {eme }}$ terme est:

$$
\left(Q_{i}-P_{i}\right) \cdot \vec{n}_{\text {Prim }_{i}}
$$

- $A_{\text {model }}$ est une matrice de dimension $\left(N_{a p p} \times 3 N_{T_{c}}\right)$. La $i^{\text {eme }}$ ligne de cette matrice contient seulement six termes non nuls adjacents à partir de l'indice $3 c^{-}\left(t_{i}\right)$ : les trois composantes de $\left(1-\alpha\left(t_{i}\right)\right) \vec{n}_{\text {Prim }_{i}}$ puis celles de $\alpha\left(t_{i}\right) \vec{n}_{\text {Primi }_{i}}$.

- $W_{\text {model }}$ est une matrice carrée diagonale de dimension $N_{\text {app }}$ et de $i^{\text {eme }}$ terme $w_{i}$.

De la même façon, selon l'équation (4) on peut écrire $E_{d e f}$ sous la forme:

$$
E_{\text {def }}(\delta)=\left\|A_{\text {def }} \delta\right\|^{2}
$$

où $A_{d e f}$ est une matrice constituée de $\left(\left(N_{T_{c}}-1\right) \times N_{T_{c}}\right)$ blocs de 3 par 3 où le bloc de coordonnées $\left(c, c^{\prime}\right)$ est $I d$ si $c^{\prime}=c$, $-I d$ si $c^{\prime}=c+1$ et nul sinon.

Enfin, d'après (10), on a l'expression matricielle:

$$
E_{r e g}(\delta)=\delta^{t} M_{r e g} \delta-2 \delta^{t} v_{r e g}+c n s t
$$

avec:

$$
\begin{gathered}
M_{r e g}=A_{\text {model }}^{t} W A_{\text {model }}+\lambda A_{\text {def }}^{t} A_{\text {def }} \\
v_{\text {reg }}=A_{\text {model }}^{t} W_{\text {model }} b_{\text {model }}
\end{gathered}
$$

C'est une forme quadratique dont le minimum est atteint pour:

$$
\nabla_{\delta} E_{r e g}=2 M_{r e g} \delta-2 v_{r e g}=0 \Leftrightarrow \delta=M_{r e g}^{-1} v_{r e g}
$$

Ce vecteur optimal $\delta$ de dimension $\left(3 * N_{T_{c}} \times 1\right)$ définit les dérives $\delta_{c}$ aux temps de contrôle $T_{c}$ associés. Par conséquent, 
on peut en déduire la nouvelle trajectoire et le nuage recalé. Le recalage par ICP se fait par itération des étapes d'appariement et de minimisation jusqu'à ce qu'un critère d'arrêt soit atteint:

$$
\left\|\delta_{c}^{t-1}-\delta_{c}^{t}\right\|<\frac{1}{100}\left\|\delta_{c}^{0}-\delta_{c}^{t}\right\|
$$

\section{Résultats et évaluation}

\subsection{Données}

Les données utilisées pour évaluer l'algorithme sont issues d'acquisitions propres à l'IGN.

Le modèle : La base de données géographiques utilisée pour cette évaluation est une partie d'un modèle 3D créé par photogrammétrie à l'aide d'images aériennes et de données cadastrales. La zone couvre un quartier du $12^{i e m e}$ arrondissement de la ville de Paris. Elle se compose d'environ 71.400 primitives géométriques triangulaires dont 25.000 correspondant aux façades.

Ce modèle est généré selon la méthode décrite dans (Durupt et Taillandier, 2006). C'est une approche de type hypothesizeand-verify où les hypothèses sont construites en énumérant des surfaces admissibles dans un arrangement de plans se basant sur des empreintes de bâtiments provenant d'une base de données 2D de l'IGN. L'arrangement choisi est alors celui qui correspond le mieux à un Modèle Numérique d'Elevation (MNE) obtenu par photogrammétrie à partir d'images aériennes. Cette approche produit le niveau de détail appelé LoD2 dans la norme CityGML: les détails sur les façades et les superstructures de toit ne sont pas modélisées. On considère que le niveau de détail du modèle est de l'ordre du mètre, pour une précision de l'ordre de $10 \mathrm{~cm}$ en planimétrie (celle de la BD 2D) et $50 \mathrm{~cm}$ en altimétrie (celle du MNE).

Les données mobiles : Le laboratoire Matis de l'IGN a développé depuis 2007 un véhicule de cartographie mobile nommé Stéréopolis II (Paparoditis et al. (2012)) pour acquérir simultanément des données image et laser géoréférencées. Ce véhicule comporte deux lasers fixes de type RIEGL qui acquièrent simultanément un nuage laser de chaque côté de la voie avec une densité de 10000 pts/s (chacun). Ces lasers couvrent un secteur angulaire de l'horizontale à $80^{\circ}$ vers le haut dans un plan orthogonal à la trajectoire. Ainsi, la plus grande partie des façades est scannée, mais pas le sol.

Les données utilisées pour l'évaluation comportent 3.6 millions de points laser s'étendant sur une distance d'environ 400 mètres et acquis en 3 minutes. Ces données sont globalement bien géoréférencées et proches du modèle $(0.5 \mathrm{~m}$ en moyenne) comme le montre la Figure 6.

$54,7 \%$ des points du nuage laser ont été sélectionnés par le descripteur géométrique utilisé (cf. Figure 6). Ces points ont une probabilité forte d'appartenir à une façade de bâtiment. Toutefois, certains de ces points peuvent correspondre à des surfaces planes verticales non présentes dans le modèle (abribus, troncs,...) comme montré en Figure 7 et qui interféreront donc de manière plus ou moins importante sur les résultats (cf. Partie 5.3).

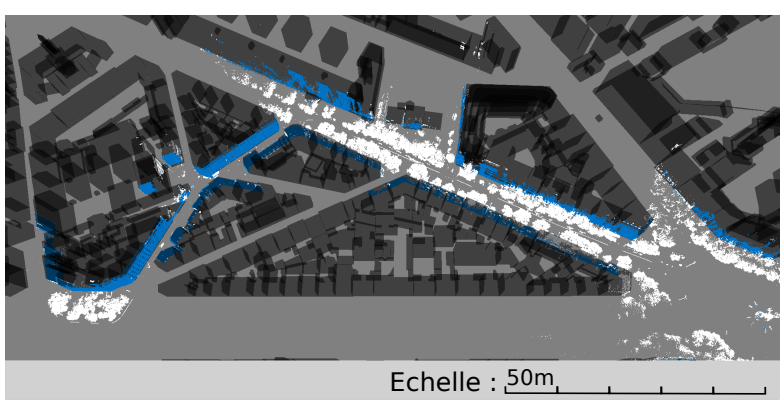

Points planaires $\square$ Points non planaires $\square$ Modèle géométrique

Figure 6: Visualisation du descripteur de planarité.

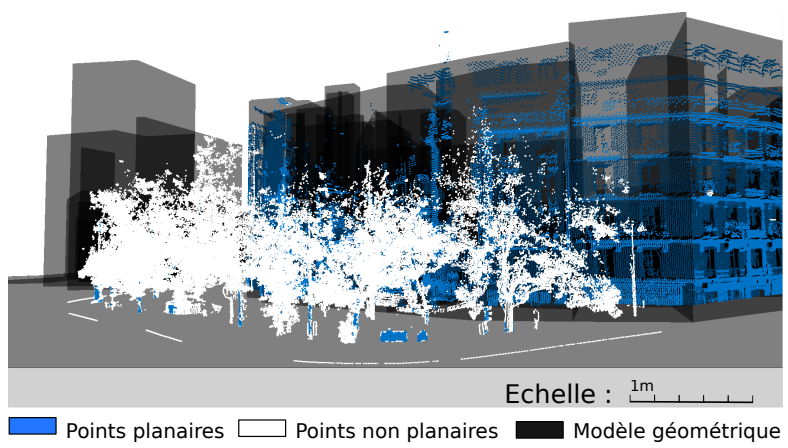

Figure 7: Illustration du score $S_{\text {facade }}$ au niveau des autres surfaces planaires verticales que les façades de bâtiments (poteaux, arbres, panneaux, ...).

\subsection{Paramétrage}

L'algorithme de recalage dépend de trois paramètres:

1. le pas de discrétisation temporelle $\Delta_{t}$,

2. le terme de rigidité $\lambda_{\text {rigid }}$,

3. la distance maximale d'appariement des points $d_{\max }$.

Ces paramètres influencent les deux caractéristiques principales d'un algorithme de recalage:

1. la robustesse: capacité de l'algorithme à recaler des données mobiles les plus éloignées possibles du modèle (cf. Partie 5.4),

2. la précision: qualité d'une telle correction (cf. Partie 5.5).

\subsubsection{Discrétisation temporelle $\Delta_{t}$}

Le pas de discrétisation temporelle $\Delta_{t}$ n'impacte que la précision, puisque la dérive réelle est approchée par une fonction linéaire par morceaux. Dans une telle approximation, l'erreur maximale sur un intervalle de temps $\Delta_{t}$ est $a \delta_{t}^{2} / 8$ où $a$ est l'accélération maximale de la dérive sur cet intervalle, c'est-à-dire l'erreur maximale sur l'accélération estimée par le système de géopositionnement. Idéalement, $\Delta_{t}$ doit être choisi pour que cette erreur soit inférieure à la précision des données. Malheureusement, il est souvent difficile d'avoir accès au $a$ pour les systèmes de géopositionnement courants car, même en connaissant la précision de la mesure accélérométrique, il est difficile d'estimer comment celle-ci est modifiée par l'intégration des autres mesures (odométrique, GPS). Pour notre système, l'erreur accélérométrique maximale mesurée expérimentalement est de l'ordre de $a=4 \cdot 10^{-3} \mathrm{~m} \cdot \mathrm{s}^{-2}$ ce 
qui donne un pas de temps $\Delta_{t}=4.47 \mathrm{~s}$. Afin d'assurer une bonne précision, même en cas de déformation beaucoup plus importante, nous avons réduit $\Delta_{t}$ à $1 s$ dans toute l'évaluation. Ce choix n'impacte que le temps de calcul de l'étape de minimisation car il accroît le nombre de temps de contrôle et par conséquent la taille des matrices à inverser. L'étape de minimisation reste négligeable face au temps d'appariement.

\subsubsection{Rigidité $\lambda_{\text {rigid }}$}

$\lambda_{\text {rigid }}$ est un paramètre adimensionnel, les deux énergies étant des sommes de carrés des distances, et les $w_{i}$ étant euxmêmes adimensionnels. Plus ce paramètre est élevé, plus il pénalise les variations de la dérive. Son réglage dépend des caractéristiques de l'acquisition. Une étude d'ordre de grandeur des énergies $E_{\text {model }}$ et $E_{\text {def }}$ montre que, pour que leurs contributions soient équivalentes, $\lambda_{\text {rigid }}$ doit être de l'ordre de:

$$
\frac{\bar{N}_{a p p}^{\Delta_{t}} d \overline{i s} t(P, P r i m)}{\Delta D^{\Delta_{t}}}
$$

où $\bar{N}_{a p p}^{\Delta_{t}}$ est le nombre moyen de points appariés par intervalle de temps $\Delta_{t}\left(10^{4}\right)$, dist $(P$, Prim $)$ est la distance moyenne des points aux primitives appariées $\left(10^{0}\right)$ et $\Delta^{-} D^{\Delta_{t}}$ est la variation moyenne de la dérive par intervalle de temps $\Delta_{t}\left(10^{-2}\right)$. Les valeurs entre parenthèses sont les ordres de grandeurs de ces valeurs pour notre système d'acquisition et donnent pour $\lambda_{\text {rigid }}$ un ordre de grandeur de $10^{6}$ que nous utiliserons lors de nos expérimentations.

Le terme de rigidité est un paramètre crucial du recalage. Nos premières expérimentations ont montré qu'il conditionne plusieurs phénomènes complexes inhérents aux données traitées. Un recalage complètement rigide $\left(\lambda_{\text {rigid }} \rightarrow \infty\right)$ ne permet pas de retrouver la dérive réelle qui est non linéaire. Par contre, une rigidité trop basse comporte deux risques majeurs:

1. Perte de cohérence globale: lorsque la dérive initiale du nuage est importante, le risque de converger vers un mauvais recalage (minimum local) augmente d'autant plus que la rigidité est faible car, dans ce cas, une partie du nuage peut facilement être appariée à de mauvaises primitives même si le reste du nuage est bien apparié. L'utilisation d'un fort terme de rigidité permet de corriger de mauvais appariements en propageant les contraintes issues des bons appariements (si ceux-ci sont majoritaires).

2. Sur-correction: comme le modèle sur lequel on recale la donnée est moins détaillé que la donnée elle même, une faible rigidité risque d'entraîner une sur-correction, c'est à dire qu'on risque de trop déformer le nuage pour "écraser" ces détails sur le modèle (cf. Figure 8).

\subsubsection{Distance maximale d'appariement $d_{\max }$ :}

Un principe central de l'ICP est de se baser sur les bons appariements pour rapprocher les données du modèle et ainsi augmenter la proportion de bons appariements jusqu'à atteindre un recalage idéal avec un maximum de bons appariements. Il n'est pas nécessaire que tous les points soient bien appariés

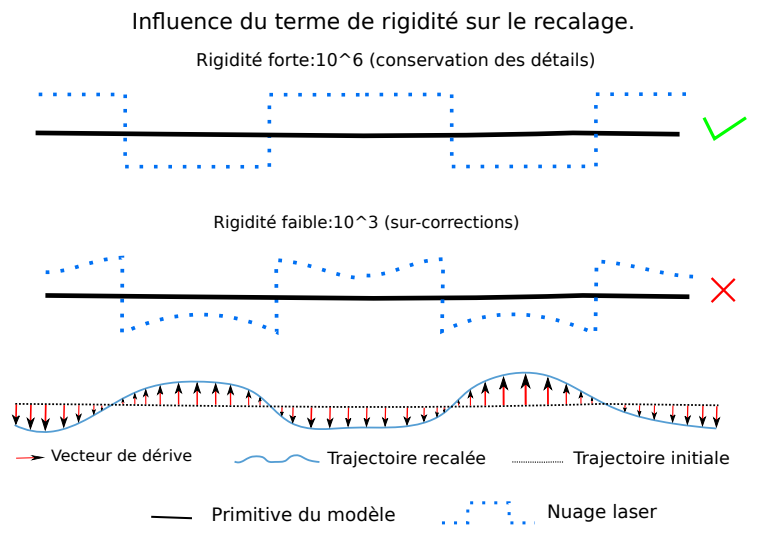

Figure 8: Phénomène de sur-correction locale: à faible rigidité, les détails absents du modèle impactent la trajectoire

dès la première itération, mais il faut qu'il y en ait suffisamment, et en suffisamment grande proportion pour que la convergence puisse démarrer. Ainsi, le paramètre $d_{\max }$ est crucial puisque s'il est choisi trop grand ou trop petit, il risque d'y avoir une trop forte proportion de mauvais appariements pour initier ce processus:

1. trop grand: tous les points seront appariés, mais souvent à tort.

2. trop petit: très peu de point seront appariés, donc des mauvais appariements accidentels auront un impact très fort.

Une bonne robustesse est ainsi obtenue en choisissant $d_{\max }$ de l'ordre de la dérive moyenne, ce qui nécessite d'en avoir une bonne estimation. Cependant, plus $d_{\max }$ est grand, plus le recalage final sera perturbé par de mauvais appariements avec des objets non modélisés (troncs d'arbres, mobiliers urbains, etc). Cela pose un problème de précision si cette distance moyenne est supérieure à la distance minimale d'objets non modélisés à la façade.

La solution que nous avons retenue est d'effectuer le recalage en deux passes successives, chaque passe étant une ICP complète, et l'initialisation de la deuxième passe étant le résultat de la première:

1. lors de la première passe, $d_{\max }=100 \mathrm{~m}$ : pour montrer la capacité de notre approche à recaler de fortes dérives, nous testerons des dérives maximales de cet ordre (bien supérieur à tout système réel). Choisir ce paramètre permet d'optimiser la robustesse dans ces conditions. Par contre, ce choix entraine une perte de précision liée aux nombreux mauvais appariements supplémentaires (cf. Figure 7).

2. lors de la seconde passe, $d_{\max }=1 \mathrm{~m}$ : ce paramètre est de l'ordre de la distance minimale d'objets non modélisés aux façades. Il permet de supprimer de nombreux mauvais appariements et d'améliorer la précision.

Les deux autres paramètres sont inchangés entre les passes.

\subsection{Résultats}

La méthode ainsi paramétrée a été utilisée pour recaler les données présentées dans la partie 5.1. A la fin de la première 

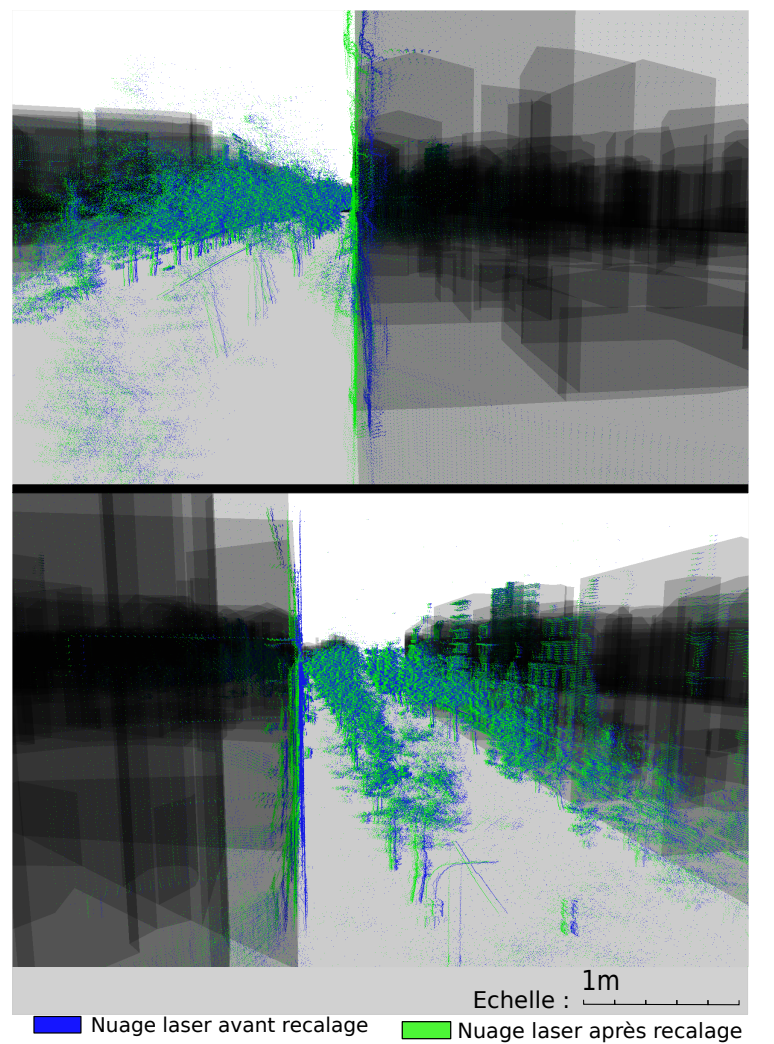

Modèle géométrique

Figure 9: Visualisation du recalage d'un nuage de points laser acquis par le véhicule Stéréopolis.

passe, 98, 7\% des points de donnée sélectionnés ont été appariés. Cette première passe diminue la distance moyenne des points aux primitives, noté $D P P$, d'environ $50 \mathrm{~cm}$ à $32 \mathrm{~cm}$. A la fin de la seconde passe, $93,89 \%$ des points sélectionnés ont été appariés et la $D P P$ passe à $9.5 \mathrm{~cm}$. Cette distance finale ne doit pas être interprétée comme une erreur de recalage. Elle vient des détails des façades scannées hors de leur plan porteur (fenêtres, balcons,...). Le modèle ayant luimême une précision de l'ordre de $10 \mathrm{~cm}$, on peut considérer ce recalage comme optimal. L'inspection visuelle du résultat obtenu (cf. Figure 9) confirme que la donnée mobile a bien été recalée sur le modèle puisque la donnée recalée est beaucoup plus proche de la façade que la donnée initiale. Ces résultats montrent l'intérêt d'utiliser différentes valeur de $d_{\max }$ lors des deux passes afin d'affiner le recalage des données. Dans notre configuration, avec une bonne initialisation, la première passe n'est pas indispensable. Cependant elle le deviendra lorsque l'on testera la robustesse de la méthode (cf. Partie 5.4).

Le recalage a duré $182 \mathrm{~s}$ au total. La première passe s'est arrêtée après 3 itérations et la seconde après 13 itérations soit environ $11 s$ par itération (appariement, résolution du système et application de la dérive). Le nombre d'itérations dépend:

1. de la distance initiale des données au modèle: plus cette distance est grande, plus il faut d'itérations pour retrouver les bons appariements.

2. du seuil utilisé comme critère d'arrêt: plus ce seuil sera faible, plus le nombre d'itérations pour l'atteindre sera élevé.

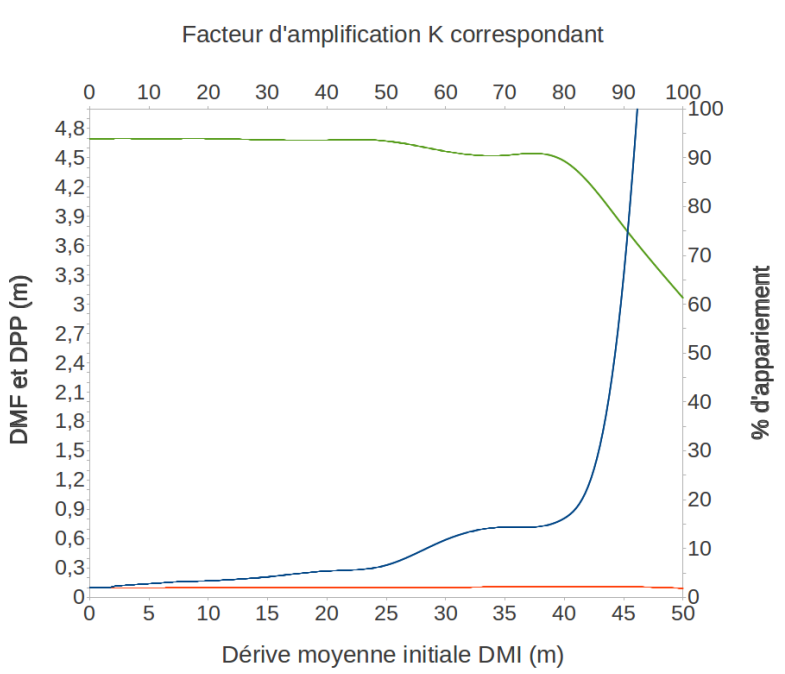

DMF - DPP - \% appariement

Figure 10: Evolution des différents indicateurs statistiques en fonction de l'éloignement à la solution.

Nous allons maintenant chercher à évaluer les performances de notre algorithme de recalage, notamment en termes de robustesse (cf. Partie 5.4) et de précision (cf. Partie 5.5).

\subsection{Robustesse}

Nous proposons de caractériser la robustesse d'un algorithme de recalage comme la dérive maximale qu'il permet de recaler correctement (même grossièrement). Cela suppose d'avoir une référence pour déterminer si le recalage est correct. Dans notre cas, le résultat de la partie précédente peut servir de référence puisque l'inspection visuelle et la distance des points aux façades permettent de le considérer comme optimal. Pour éviter de devoir définir explicitement ce qu'est un recalage "correct", nous allons illustrer la robustesse par une courbe indiquant la dérive moyenne finale $(D M F)$ ) (après les deux passes de recalage) en fonction de la dérive moyenne initiale $(D M I)$. Ces dérives moyennes sont simplement la distance $D M$ définie dans l'équation (3) calculée respectivement entre le nuage final et la référence, et entre le nuage initial et la référence.

Pour tracer cette courbe, nous allons amplifier la dérive réelle estimée (cf. Partie 5.3) pour générer des données mobiles $A_{K}$ synthétiques de plus en plus éloignées de la référence:

$$
A_{K}=A_{\text {ref }}+K\left(A_{\text {ori }}-A_{\text {ref }}\right)
$$

où $A_{\text {ori }}$ est la concaténation de tous les points de la donnée mobile originale (cf. Partie 1.3), $A_{\text {ref }}$ celle de la donnée recalée (cf. Partie 5.3) et $K$ le facteur d'amplification, de sorte que $A_{0}=A_{\text {ref }}$ et $A_{1}=A_{\text {or } i}$. La dérive moyenne de notre donnée originale étant de $0,5 \mathrm{~m}$, la dérive moyenne d'un nuage généré $A_{K}$ sera donc de $K \times 0.5 \mathrm{~m}$.

Ainsi, nous avons généré et recalé 16 nuages pour des valeurs $K$ allant de 1 à 100 . Les résultats sont illustrés dans la Figure 10. Sont illustrés la dérive moyenne finale ( $D M F$, bleu), mais aussi le pourcentage de points appariés (vert) et la distance moyenne des points appariés aux primitives ( $D P P$, rouge) en fonction de la dérive moyenne initiale. 


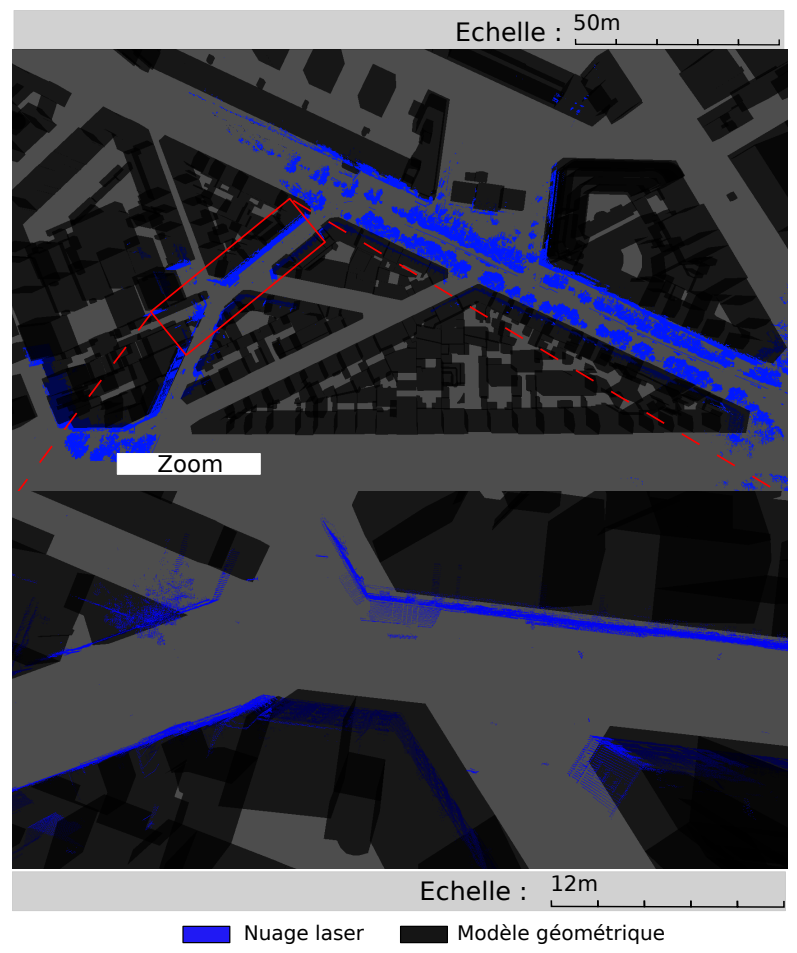

Figure 11: Visualisation d'un nuage de points laser éloigné d'une distance moyenne de 2,5 mètres à la solution.

La courbe bleue $(D M F)$ rend compte de la robustesse. Elle montre que l'algorithme parvient à recaler les nuages de points générés de façon grossière (moins d'1 $\mathrm{m}$ ) jusqu'à un facteur d'amplification $K$ de $80(D M I=40 \mathrm{~m})$. Un exemple de ce recalage est montré dans les Figures 11 et 12 correspondant au cas $K=5(D M I=2.5 \mathrm{~m})$. Pour des amplifications supérieures à $80(D M I>40 \mathrm{~m})$, la $D M F$ explose, ce qui indique que le recalage échoue. Plus précisément, l'ICP converge vers un minimum local à cause de trop nombreux mauvais appariements. Un exemple d'un tel recalage est montré dans les Figures 13 et 14 correspondant au cas $K=100$ $(D M I=50 \mathrm{~m})$

La courbe rouge de la Figure 10 représente la Distance des Points aux Primitives appariées $(D P P)$ pour chaque nuage recalé. Elle reste très faible (de l'odre de $10 \mathrm{~cm}$ ) quelle que soit l'amplification, ce qui montre que l'énergie $E_{\text {model }}$ est toujours bien minimisée par l'ICP, que le recalage soit bon ou pas, c'est-à-dire que les points appariés sont toujours proches du modèle (mais pas toujours des bonnes primitives).

La courbe verte de la Figure 10 représente le pourcentage de points appariés à la fin du recalage. Elle montre que la limite à $K=80$ correspond à un moment où une trop forte proportion des points ne sont plus appariés.

Dans le cas limite $(K=80)$, le recalage fait passer la déformation moyenne de $40 \mathrm{~m}$ à $81 \mathrm{~cm}$. Cette valeur est limitée en terme de précision mais elle reste très satisfaisante au vu de l'éloignement de l'initialisation de départ.

Ces résultats démontrent que notre processus permet de recaler des nuages de points sur un modèle avec une initialisation bien plus mauvaise que celle fournie par notre système de géopositionnement, et est donc potentiellement applicable à la plupart des systèmes existants, puisque, en reprenant nos

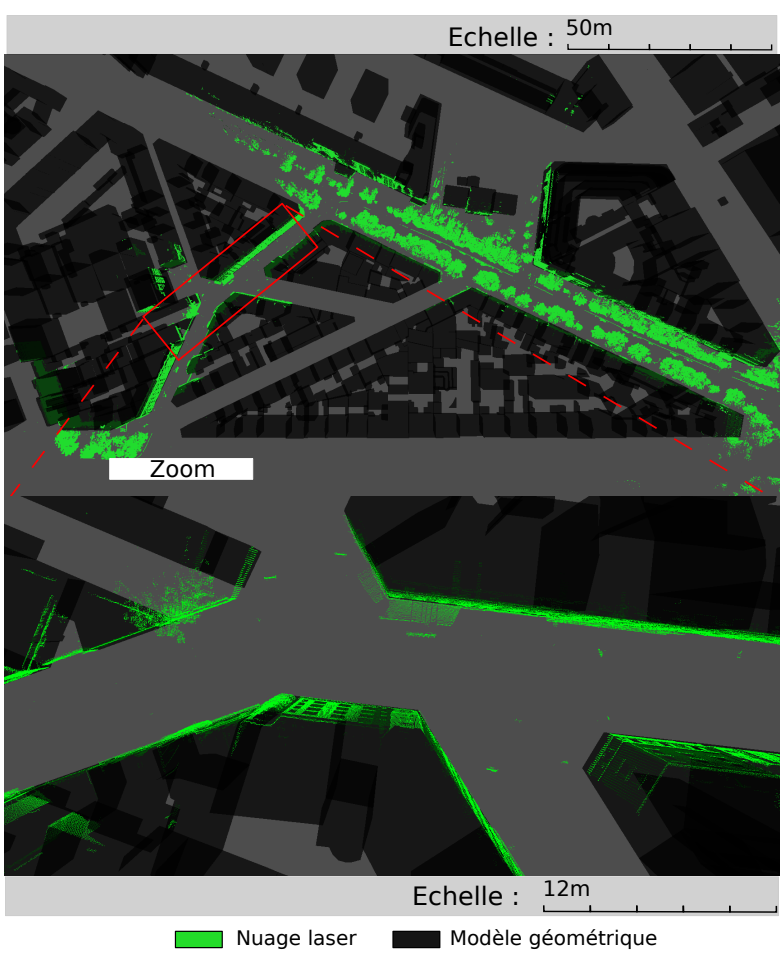

Figure 12: Visualisation de ce nuage de points laser après recalage.

hypothèses:

- lenteur de la variation de la dérive: notre évaluation montre une robustesse à des amplitudes de dérive relativement élevées,

- qualité de l'orientation: cette hypothèse est la plus contraignante et nous proposons en perspective (cf.Partie 6.2) des pistes pour s'en affranchir,

- dérive indépendante de la vitesse du véhicule: ceci est vrai de tous les systèmes que nous connaissons.

La robustesse de notre méthode à des dérives nettement supérieures à celle de notre système indique que celle-ci pourra donc être appliquée pour des données provenant de véhicules de cartographie mobile équipés de capteurs moins performants (et donc moins coûteux).

Nous allons maintenant évaluer la précision de notre recalage.

\subsection{Précision}

La précision est l'autre qualité importante d'une méthode de recalage. Elle impacte directement ses applications. Cependant, la notion de précision est difficile à définir dans notre contexte puisque les données que nous recalons sont de nature et de niveau de détails très différents, et que notre référence n'a elle même qu'une précision limitée (de l'ordre de $10 \mathrm{~cm}$ ).

\subsubsection{Première définition}

On peut proposer une première définition de la précision en se basant sur l'évaluation de la robustesse, et en particulier, la courbe bleue de la Figure 10. Pour des valeurs de Dérive Moyenne Initiale $(D M I)$ inférieures à $25 m$ (cas très bien recalés), cette courbe montre une tendance linéaire $D M F \approx$ 


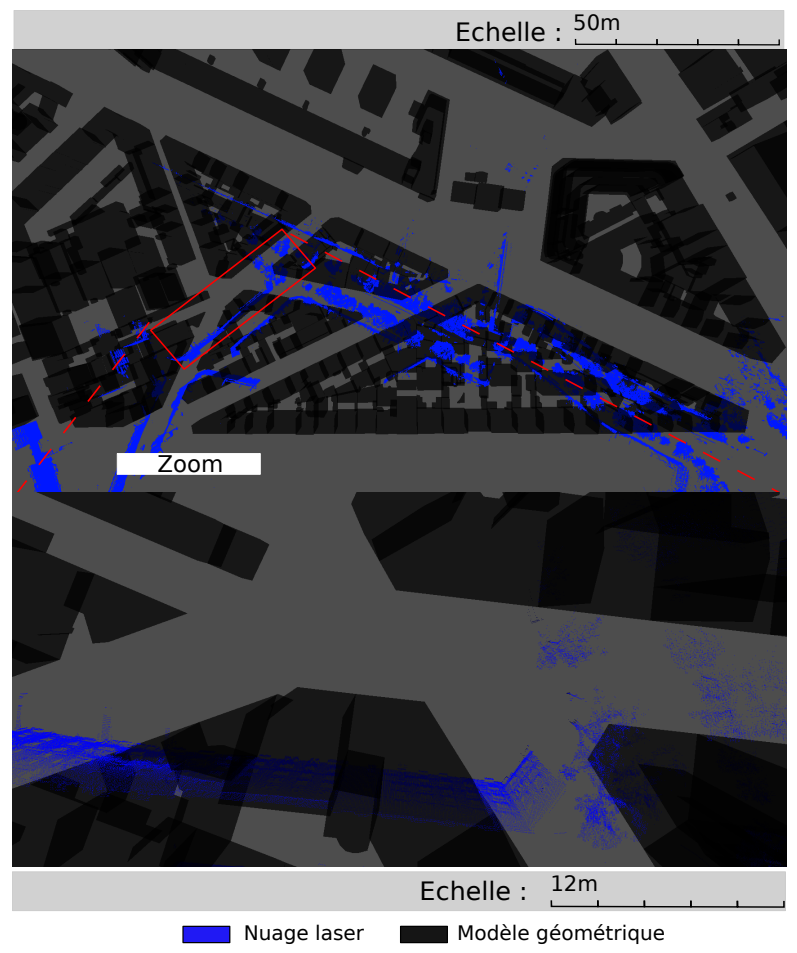

Figure 13: Visualisation d'un nuage de points laser éloigné d'une distance moyenne de 50 mètres à la solution.

$D M I / 75$, c'est-à-dire que le recalage rapproche environ 75 fois la donnée d'entrée de la référence. Cela montre que la précision n'est pas absolue mais dépend de l'initialisation, et qu'un "facteur d'amélioration" est sans doute la meilleure caractérisation de la précision que l'on puisse donner. Ce problème ne se pose pas en recalage rigide car l'énergie minimisée ne dépend pas de l'entrée (à appariements identiques), alors que notre terme de rigidité, lui, en dépend. Cette première définition de la précision est tout de même biaisée car:

1. elle dépend de la référence, qui a été créée en appliquant ce recalage pour $K=1$ donc peut présenter des défauts similaires aux cas amplifiés $K>1$,

2. elle ne correspond pas à la qualité la plus importante pour les applications qui est la répétabilité du recalage, c'est-à-dire que les recalages de données laser issues d'acquisitions différentes sur un même modèle doivent être proches entre eux.

\subsubsection{Deuxième définition}

Nous allons proposer une deuxième définition qui ne souffre pas de ces deux limites en mesurant la variabilité des résultats du recalage en fonction de la variabilité des données de départ. Autrement dit, nous allons essayer d'identifier si notre recalage est capable de donner le même résultat avec des données initiales différentes. Contrairement à l'évaluation de la robustesse, cela va nécessiter de générer tout un ensemble de données ayant une Dérive Moyenne Initiale $(D M I)$ proche mais avec des dérives différentes (simulant des dérives réelles).

Pour effectuer cette évaluation, nous avons simulé des dérives en intégrant deux fois un bruit gaussien de variance $\sigma$ avec la contrainte d'une dérive nulle à chaque extrémité de la trajectoire. Ce choix simule un masque GPS de la durée

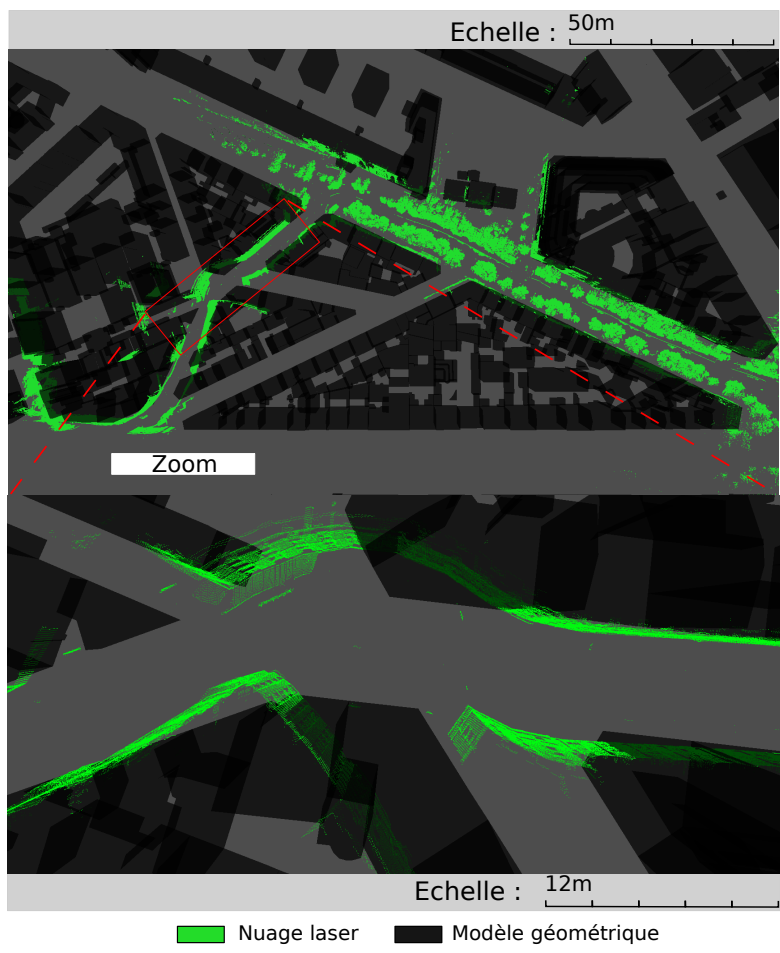

Figure 14: Visualisation de ce nuage de points laser après recalage.

de notre acquisition ( 3 minutes) pour lequel une position n'est connue qu'au début et à la fin, et pour lequel l'information accélérométrique (plus ou moins précise) est utilisée pour interpoler la trajectoire entre ces points. La valeur de sigma $\sigma$ modélise la précision de l'information accélérométrique et définit la DMI des nuages créés. Plus cette valeur augmente, plus les nuages peuvent avoir une DMI importante. Pour une valeur de $\sigma$ donnée, nous avons généré 20 dérives pour créer 20 données mobiles tests différentes ayant une DMI très proche à partir de notre propre donnée recalée. Ceci a été réalisé pour quatre valeurs de $\sigma$ soit un total de 80 nuages tests.

La variabilité des données d'entrée et la variabilité des données de sortie seront définies comme la moyenne des distances entre les dérives estimées sur toutes les paires $1 \leq i<j \leq 20$ totalisant $\frac{(20 * 19)}{2}=190$ paires différentes possibles pour chaque $\sigma:$

$$
D M_{i}^{j}=\frac{1}{N_{T_{c}}} \sum_{c=1}^{N_{T_{c}}}\left\|\delta_{c}^{j}-\delta_{c}^{i}\right\|
$$

Étant donné que nous évaluons ici la précision et non la robustesse, les nuages ont été choisis de sorte à ce que le recalage soit toujours correct.

\subsubsection{Résultats}

Les résultats de cette évaluation sont présentés sur la Figure (15). Ce graphique représente la variabilité moyenne calculée sur tous les couples possibles en fonction de la Dérive Moyenne Initiale DMI (ou du coefficient $\sigma$ utilisé).

La courbe rouge représente la variabilité moyenne calculée sur les 20 nuages avant le recalage pour chaque $\sigma$. En prenant l'exemple suivant, $\sigma=0.16$, l'interprétation de cette courbe est la suivante: pour cette valeur de $\sigma$, les nuages ont été générés avec une DMI de $22 m$ ce qui signifie que les nuages 
sont globalement initialisés loin de notre solution (déduite visuellement dans les parties précédentes), et ils ont, entre eux, une variabilité initiale moyenne d'environ $20 \mathrm{~m}$ traduisant le fait que les dérives appliquées sont bien aléatoires (les nuages sont loin d'être identiques).

La courbe bleue montre cette variabilité moyenne après recalage et l'interprétation de la courbe se fait de la même manière. Elle montre une dépendance linéaire entre la DMI et les variabilités d'entrée et de sortie, avec un facteur légèrement inférieur à 0.05 . Cela montre que le recalage réduit la variabilité des données d'un facteur proche de 5 quel que soit le coefficient $\sigma$ utilisé. Nous savons que tous ces recalages sont bons (bonne convergence de l'ensemble) mais ce facteur d'amélioration (reproductibilité de la réponse) est faible. Ce faible coefficient d'amélioration est en partie dû au paramètre de rigidité $\lambda_{\text {rigid }}$ utilisé. Plus celui-ci est élevé, plus les nuages ont du mal à se déformer car le terme de rigidité dans le processus de minimisation devient prépondérant au détriment du terme d'attache au modèle. Par conséquent les résultats finaux ne pourront être identiques.

Ainsi, nous avons décidé d'effectuer la même expérience en changeant le paramètre $\lambda_{\text {rigid }}$ lors de la seconde passe (passant de $10^{6}$ à $10^{4}$ ). Les résultats obtenus sont visibles sur la courbe verte. Cette courbe montre qu'en diminuant la rigidité, la variabilité de la réponse diminue. En diminuant d'un facteur 100 la rigidité, nous avons gagné un facteur 2 en précision. Ces deux courbes (bleue et verte) attestent de l'importance du choix de $\lambda_{\text {rigid }}$ dans le résultat du recalage. Cela souligne qu'un compromis est à réaliser entre la robustesse de l'algorithme aux déformations et sa précision. Cela montre également que le recalage des données avec un paramétrage adéquat réduit la variabilité des données d'un facteur supérieur à 10 quelque soit le coefficient $\sigma$ utilisé.

Ce facteur est plus faible que celui auquel on pouvait s'attendre dans l'évaluation de robustesse où la dérive moyenne était divisée par 75 dans certains cas. Plusieurs explications sont possibles. D'une part, nos simulations ont tendance à générer des dérives dont les variations ont des fréquences plus élevées que celle de notre système. D'autre part, la référence utilisée était biaisée (elle était suffisante pour qualifier la robustesse mais pas la précision). L'inspection visuelle de ces nouveaux résultats montre que l'erreur de précision provient majoritairement de translations parallèles aux façades sur lesquelles nous n'avons pas de contraintes directes. En l'absence de contraintes dans une direction, le terme de rigidité est prépondérant, ce qui conduit à une interpolation linéaire de la dérive (dans cette direction) entre les points qui ont euxmêmes des contraintes dans cette direction. Toute la partie non linéaire de la dérive (dans cette direction) entre ces points ne peut donc pas être estimée. Dans le cas d'une amplification de dérive, ces translations parallèles sont estimées de façon proche, ce qui n'est plus le cas avec les dérives plus aléatoires générées ici. Ces translations parallèles sont donc la limite majeure à la précision, puisque le long d'une rue avec des façades toutes parallèles, on ne peut se reposer que sur la propagation de contraintes par la rigidité pour estimer les translations parallèles. La précision est donc principalement limitée par ce facteur de rigidité et ce manque d'information parallèlement aux façades.

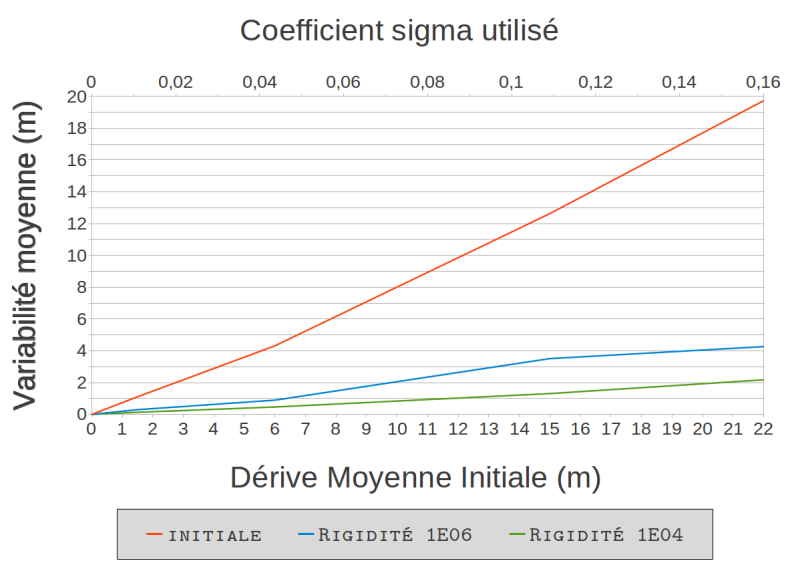

Figure 15: Variabilité obtenue sur tout les couples testés pour un epsilon donné.

\section{Conclusion et perspectives}

\subsection{Conclusion}

Le but de notre démarche était de définir un processus capable de recaler des données laser mobiles sur un modèle géométrique en prenant en compte la non-linéarité des déformations en fonction du temps. Un algorithme de recalage basé sur le principe de l'ICP (Iterative Closest Point) de point à surface et un modèle de déformation linéaire par morceaux en fonction du temps ont été mis en place. Les résultats obtenus sont très satisfaisants puisque l'évaluation de la robustesse montre que l'algorithme peut recaler des dérives jusqu'à 80 fois supérieures à celles de notre système ce qui donne une forte garantie sur son utilisabilité. En termes de précision, le bilan est plus mitigé car:

- d'une part, l'inspection visuelle ne permet pas de distinguer le résultat du recalage des données de notre système d'un résultat "optimal".

- d'autre part, le recalage ne fait gagner qu'un facteur 10 sur la précision des données sur des trajectoires simulées de façon aléatoires.

La méthode de recalage décrite dans cet article est facile à paramétrer puisqu'elle n'a que deux paramètres influents: le terme de rigidité $\lambda_{\text {rigid }}$ et la distance maximale d'appariement $d_{\text {max }}$ qui sont tous deux simples à interpréter.

La méthode est aussi très générique puisqu'elle est applicable tant aux modèles en 2D que 3D, et que les hypothèses de travail sur la dérive réelle ne sont pas trop limitantes, la plus contraignante étant la précision de l'orientation.

Enfin, l'algorithme proposé est rapide puisqu'il permet de recaler en $182 s$ une donnée acquise en 3 minutes. Dans la perspective d'un passage en production, nous pouvons cependant espérer traiter pendant la nuit les données acquises pendant la journée.

\subsection{Perspectives}

Pour aller encore plus loin, nous pourrions envisager de :

- trouver des schémas de décroissance appropriés pour les deux paramètres $\lambda_{\text {rigid }}$ et $d_{\max }$ afin d'améliorer à la 
fois la robustesse, la précision et le temps de calcul (par l'intermédiaire du nombre d'itérations). Une approche optimale passerait sûrement par une décroissance progressive des deux paramètres: les valeurs fortes au début assurant une bonne robustesse en recalant uniquement les basses fréquences et les valeurs faibles à la fin améliorant la précision en recalant des fréquences plus hautes. Cela sera d'autant plus aisé que l'utilisateur donnera un ordre de grandeur de la dérive réelle.

- étudier des dérives plus réalistes que nos dérives simulées aléatoires mais moins spécifiques que nos amplifications de la dérive réelle. Cela pourrait être réalisé en faisant une étude plus poussée des propriétés statistiques des dérives des systèmes de géopositionnement existants et en générant des déformations aléatoires ayant ces propriétés statistiques.

- utiliser une méthode de minimisation autre que la méthode par moindres carrés pour donner moins d'importance aux mauvais appariements, ou pondérer les appariements en fonction de la distance point/primitive afin d'améliorer la précision sans réduire la robustesse.

- utiliser la trajectoire résultant du recalage pour réestimer l'orientation du véhicule afin de s'affranchir de la limite majeure d'utilisabilité actuelle (précision de l'orientation). La trajectoire permet en effet d'estimer l'un des vecteurs du référentiel véhicule (celui pointé vers l'avant qui doit tangenter la trajectoire), une inconnue restant sur la rotation autour de ce vecteur. Les routes urbaines étant peu déversantes, l'erreur d'estimation de cette dernière rotation sera sûrement négligeable.

- utiliser les informations présentes dans les images.

\section{Références}

Al-Durgham, M., Detchev, I., Habib, A., 2010. Analysis of two triangle-based multi-surface registration algorithms of irregular point clouds. ISPRS Commission V.

Barnea, S., Filin, S., 2008. Keypoint based autonomous registration of terrestrial laser point-clouds. ISPRS Journal of Photogrammetry and Remote Sensing 63 (1), 19-35.

Besl, P., McKay, N., 1992. A method for registration of 3-d shapes. IEEE Transactions on Pattern Analysis and Machine Intelligence 14 (2), 239-256.

Chen, C., Hung, Y., Cheng, J., 1999. Ransac-based darces: a new approach to fast automatic registration of partially overlapping range images. IEEE Transactions on Pattern Analysis and Machine Intelligence 21 (11), 1229-1234.

Chen, Y., Medioni, G., 1992. Object modelling by registration of multiple range images. Image and Vision Computing 10 (3), 145-155.

Demantké, J., Vallet, B., Paparoditis, N., 2012. Streamed vertical rectangle detection in terrestrial laser scans for facade database production. AnnalsPRS I-3 (2012), 99-104.

Dold, C., Brenner, C., 2006. Registration of terrestrial laser scanning data using planar patches and image data. IAPRS 36 (Part 5), 7883.

Durupt, M., Taillandier, F., 2006. Automatic building reconstruction from a digital elevation model and cadastral data: An operational approach. PCV.
Eggert, D., Dalyot, S., 2012. octree-based simd strategy for icp registration and alignment of $3 \mathrm{~d}$ point clouds. ISPRS III.

Fitzgibbon, A., 2003. Robust registration of $2 \mathrm{~d}$ and $3 \mathrm{~d}$ point sets. Image and Vision Computing 21 (13-14), 1145-1153.

Gelfand, N., 2005. Robust global registration. Proceedings of the third Eurographics symposium on Geometry processing 38 (2), 197.

Gelfand, N., Ikemoto, L., Rusinkiewicz, S., Levoy, M., 2003. Geometrically stable sampling for the icp algorithm. Fourth International Conference on 3D Digital Imaging and Modeling 2003 3DIM 2003 Proceedings 0, 260-267.

Gressin, A., Mallet, C., Demantké, J., David, N., 2013. Towards 3d lidar point cloud registration improvement using optimal neighborhood knowledge. IJPRS.

Hahnel, D., Thrun, S., Burgard, W., 2003. An extension of the icp algorithm for modeling nonrigid objects with mobile robots. International Joint Conference on Artificial Intelligence 18 (1), 915-920.

Hecker, Y., Bolle, R., 1994. On geometric hashing and the generalized hough transform. Ieee Transactions On Systems Man And Cybernetics 24 (9), 1328-1338.

Huber, D., Hebert, M., 2003. Fully automatic registration of multiple $3 \mathrm{~d}$ data sets. Image and Vision Computing 21 (7), 637-650.

Jian, B., Vemuri, B., 2005. A robust algorithm for point set registration using mixture of gaussians. Proceedings IEEE International Conference on Computer Vision IEEE International Conference on Computer Vision 2 (II), 1246-1251.

Johnson, A., Hebert, M., 1999. Using spin images for efficient object recognition in cluttered 3d scenes. IEEE Transactions on Pattern Analysis and Machine Intelligence 21 (5), 433-449.

Paparoditis, N., Papelard, J.-P., Cannelle, B., Devaux, A., Soheilian, B., David, N., Houzay, E., 2012. Stereopolis II: A multi-purpose and multi-sensor $3 \mathrm{~d}$ mobile mapping system for street visualisation and 3d metrology. Revue Francaise de Photogrammétrie et de Télédétection 200, 69-79.

Pottmann, H., Leopoldseder, S., Hofer, M., 2004. Registration without icp. Computer Vision and Image Understanding 95 (1), 54-71.

Ripperda, N., Brenner, C., 2005. Marker free registration of terrestrial laser scans using the normal distribution transform. International Archives of the Photogrammetry, Remote Sensing and Spatial Information Sciences XXXVI-5/W17 (on CD-ROM).

Rusinkiewicz, S., Levoy, M., 2001. Efficient variants of the icp algorithm. Proceedings Third International Conference on 3D Digital Imaging and Modeling pages, 145-152.

Salvi, J., Matabosch, C., Fofi, D., Forest, J., 2007. A review of recent range image registration methods with accuracy evaluation. Image Vision Comput. 25 (5), 578-596.

Sharp, G., Lee, S., Wehe, D., 2002. Icp registration using invariant features. IEEE Transactions on Pattern Analysis and Machine Intelligence 24 (1), 90-102.

Stein, F., Medioni, G., 1992. Structural indexing: Efficient 3-d object recognition. IEEE Transactions on Pattern Analysis and Machine Intelligence 14 (2), 125-145.

Tarel, J.-P., Boujemaa, N., 1995. Une approche floue du recalage 3d: généricité et robustesse. Rapport de recherche no 2716.

Thirion, J.-P., 1996. New feature points based on geometric invariants for $3 \mathrm{~d}$ image registration. International Journal of Computer Vision 18 (2), 121-137.

Tsin, Y., Kanade, T., 2004. A correlation-based approach to robust point set registration. Computer VisionECCV 2004 0, 558-569.

Wolfson, H. J., Rigoutsos, I., 1997. Geometric hashing: an overview. IEEE Computational Science and Engineering 4 (4), 10-21.

Zhang, Z., 1994. Iterative point matching for registration of freeform curves and surfaces. International Journal of Computer Vision 13 (2), 119-152.

Zheng, Q., Sharf, A., Tagliasacchi, A., Chen, B., Zhang, H., Sheffer, A., Cohen-Or, D., 2010. Consensus skeleton for non-rigid spacetime registration. Computer Graphics Forum 29 (2), 635-644. 